\title{
The role of close pair interactions in triggering stellar bars and rings
}

\author{
Preethi Nair ${ }^{1}$, Sara Ellison ${ }^{2}$ and David Patton ${ }^{3}$ \\ ${ }^{1}$ Space Telescope Science Institute, Baltimore, MD 21218 USA, email: nair@stsci.edu \\ ${ }^{2}$ University of Victoria \\ ${ }^{3}$ Trent University
}

\begin{abstract}
Recent works which have looked at bars in clusters versus the field have found no significant difference in bar fraction. However, other works (Nair \& Abraham 2010, Lee et al. 2012) have found that bar fractions depend sensitively on the mass, morphology and color of the galaxy. In addition, simulations suggest that bar formation may depend on the merger ratio of close pair interactions as well as on the separation between the pairs. In this work, we analyze the bar fractions in a complete sample of $\approx 23,000$ close pairs derived from the Sloan Digital Sky Survey Data Release 7. We will present results illustrating the dependence of bar and ring fractions as a function of merger mass ratio, pair separation, galaxy morphology, and stellar mass. I will further compare the role of bars and close pairs in triggering central star formation and AGN.
\end{abstract}

\section{References}

Nair, P. \& Abraham, R. G. 2010, ApJ 714, L260

Lee, J. H., et al. 2012, ArXiv 1211.3973 